\author{
C. J. Newman · R. N. Laurini · Y. Lesbros \\ O. Reinberg · B. J. Meyrat
}

\title{
Interstitial cells of Cajal are normally distributed in both ganglionated and aganglionic bowel in Hirschsprung's disease
}

Accepted: 10 December 2002/Published online: 18 October 2003 (C) Springer-Verlag 2003

\begin{abstract}
Surgery for Hirschsprung's disease is often complicated by post-operative bowel motility disorders. The impact of intestinal neural histology on the surgical outcome has been previously studied, but no information is available concerning the influence of the distribution of interstitial cells of Cajal (ICC) on these complications. These cells are considered to be pacemakers in the gastrointestinal tract. The aim of this study was to assess the distribution of ICC in the proximal segment of resected bowel in Hirschsprung's disease and confront these results with the clinical outcome. Using immunohistochemistry for light microscopy, we compared the pattern of distribution of ICC in the proximal segment of resected bowel in Hirschsprung's disease with that in normal colon. We correlated these results with the corresponding neural intestinal histology determined by CD56 and the protein gene product 9.5 immunohistochemistry. The distribution of ICC in the proximal segment of resected bowel is identical to that of normal colon, regardless of normal or abnormal colon innervation. ICC distribution does not seem to contribute to post-operative bowel motility disorders in patients operated for Hirschsprung's disease
\end{abstract}

Keywords Interstitial cells of Cajal · Hirschsprung's disease · Post-operative complications · Enteric nervous system

C. J. Newman · Y. Lesbros · O. Reinberg · B. J. Meyrat ( $₫)$

Department of Pediatric Surgery,

Centre Hospitalier Universitaire Vaudois (CHUV),

1011 Lausanne, Switzerland

E-mail: blaise-julien.meyrat@chuv.hospvd.ch

Fax: + 41-21-3143076

R. N. Laurini

University Institute of Pathology,

Centre Hospitalier Universitaire Vaudois (CHUV),

Lausanne, Switzerland

\section{Introduction}

Bowel motility disorders are a common occurrence after pull-through surgery for Hirschsprung's disease. These mainly manifest by constipation, overflow incontinence and enterocolitis bouts. Abnormalities of the innervation of the remaining colon $[9,20]$ and transition-zone pull-through [2] have been reported as important factors contributing to these disorders.

Interstitial cells of Cajal (ICC) are considered to be pacemaker cells that generate physiological slow waves in the gastrointestinal tract and have an important role in the control of gut motility [22, 6, 11, 23]. Abnormal distribution or a lack of these cells in association with Hirschsprung's disease [28, 25, 5] and allied Hirschsprung's disorders [29, 7] have been previously described. The possibility of abnormal distribution of ICC in the remaining colon after pull-through surgery has not been previously examined.

The aim of our study was to determine the topography of ICC in the proximal segment of resected bowel in Hirschsprung's disease by c-Kit immunohistochemistry for light microscopy. The innervation of the corresponding segment was studied by CD56 and protein gene product 9.5 (PGP 9.5) immunohistochemistry to detect associated neuronal disorders $[10,14,26,16,12]$.

\section{Materials and methods}

We operated 65 patients in our institution for Hirschsprung's disease between 1977 and 1999. Paraffin-embedded tissues were available for immunohistochemistry in 48 patients. In these 48 patients (37 boys, 11 girls; 3.4:1 male:female ratio) full-thickness specimens of the proximal segment of resected bowel were obtained during definitive surgery for Hirschsprung's disease. Patient ages ranged from 2 months to 9 years (mean 26 months, SD 24 months) at the time of surgery. The patients were operated according to Duhamel's colon pull-through technique. Their post-operative follow-up ranged from 1 month to 11 years (mean 29 months, SD 32 months). Post-operative bowel motility disorder rates were obtained on the basis of a retrospective review of the patients' 
medical files. Disorders included enterocolitis bouts, persistent constipation defined as constipation persisting for a year or more after surgery and requiring medical treatment (oral laxatives or enemas), and overflow incontinence defined as incontinence episodes occurring at least once a week.

Control specimens were obtained from 16 children (12 boys, 4 girls; 3:1 male:female ratio). Ages ranged from 20 months to 15 years (mean 70 months, SD 42 months). They were operated in our institution for caustic oesophageal stenosis by oesophageal replacement with transverse colon interposition, during which fullthickness colon biopsies were taken at the proximal extremity of the transverse colon transplant (hepatic flexure) in nine children and at the distal extremity (splenic flexure) in seven. None of these children had a previous history of bowel motility disorders.

The enteric nervous system was assessed by immunohistochemistry for CD56, a killer-cell-associated antigen with expression comparable to that of neural cell adhesion molecule (NCAM) [14, 16,12 ], and for the protein gene product 9.5 (PGP 9.5), a cytoplasmic marker specific for the neural system $[10,14,26]$. This combination of cell membrane and cytoplasmic immunostaining is routinely used in our institution for intestinal innervation assessment and yields equal to superior quality results when compared with neuron-specific enolase, neurofilament protein 100 and microtubule-associated protein immunohistochemistry. ICC distribution was assessed by c-Kit immunohistochemistry. No other markers are known for ICC immunostaining.

All serial histological sections were viewed by a senior pathologist. He applied the criteria specified by Meier-Ruge [20, 4, 13] for the diagnosis of aganglionosis, intestinal neuronal dysplasia type $\mathrm{B}$ (IND B), hypoganglionosis and other dysganglionoses. He assessed ICC distribution in each layer semi-quantitatively ( 0 : absent ICC, - : diminished concentration, + : normal concentration, ++ : high concentration) by comparison with control specimens. Proximal segment innervation was compared with the bowel function outcome.

\section{Histological techniques}

The specimens were fixed in buffered formalin (4\% paraformaldehyde solution in phosphate buffered saline), dehydrated and embedded in paraffin wax. Paraffin sections $(6 \mu \mathrm{m}$ thick) were cut and mounted on 3-aminopropyl-triethoxysilane-coated glass slides, deparaffinised in xylol, immersed for $45 \mathrm{~min}$ in methanol with $0,3 \%$ hydrogen peroxide to block endogenous peroxidase and rehydrated through graded alcohols. Sections were then subjected to microwave pre-treatment in $10 \mathrm{mM}$ citrate buffer (pH 6) for $20 \mathrm{~min}$, according to the method of Shi et al. [21] and rinsed in Tris-buffered saline (TBS: $0.05 \mathrm{M}$ Tris, $0.9 \%$ sodium chloride, $\mathrm{pH}$ 7.6). Consecutive sections were then labeled for CD56, PGP 9.5 and c-Kit (Fig. 1)

For CD56 immunodetection, the first antibody used was antiCD56 monoclonal antibody (1:50, CD56, clone 1B6; Novocastra, Newcastle upon Tyne, UK) and the second biotinylated horse antimouse IgG (1:200, Vector, Burlingame, USA). For PGP $9.5 \mathrm{im}-$ munodetection, the first antibody used was rabbit anti-PGP 9.5 polyclonal antibody (1:800, Protein Gene Product 9.5, Anawa, Wangen, $\mathrm{CH}$ ) and the second biotinylated goat anti-rabbit $\mathrm{IgG}$ (1:400, Vector Laboratories, Burlingame, CA. USA). For c-kit immunodetection, the first antibody used was rabbit anti-c-kit polyclonal antibody (1:100, c-kit, MBL, Nagoya, Japan) and the second biotinylated goat anti-rabbit $\operatorname{IgG}$ (1:400, Vector Laboratories, Burlingame, CA. USA).

To reduce non-specific antibody binding, the sections were incubated in normal goat serum for c-kit and PGP 9.5 and normal horse serum for NCAM (1:30 in TBS) for $10 \mathrm{~min}$, then the first antibody mixed in TBS containing 5\% non-fat dried milk for $30 \mathrm{~min}$ at room temperature. They were then washed in TBS and incubated with the second antibody for $60 \mathrm{~min}$ at room temperature. After washing in TBS, sections were incubated in avidin-biotin-horseradish peroxidase complex according to the manufacturer's indications (Vectastain ABC kit, Vector

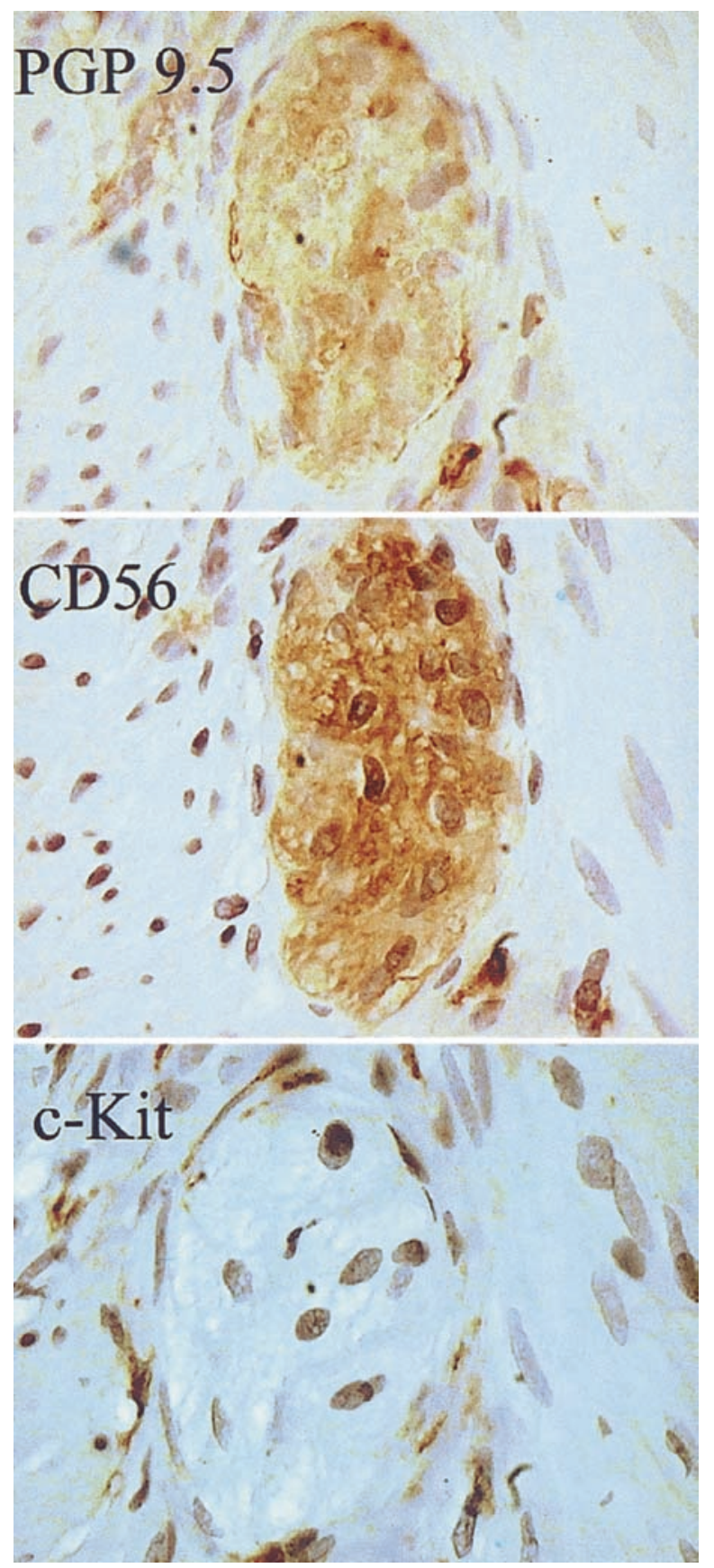

Fig. 1 Serial sections of a myenteric plexus ganglion in a normal colon segment. Ganglion cells are identified by CD56 and PGP 9.5 immunohistochemistry and interstitial cells of Cajal (ICC) by c-Kit immunohistochemistry (PGP 9.5, CD56 and c-Kit immunohistochemistry, $\times 400$ )

Laboratories, USA). Peroxidase was visualised by freshly prepared 3,3'-Diaminobenzidine-tetrahydrochloride dihydrate (DAB, Fluka Chemie, Buchs, Switzerland), $50 \mathrm{mg}$ in $100 \mathrm{ml}$ TBS containing $0.01 \%$ of hydrogen peroxide. After washing with water, the tissue sections were counterstained in Mayer's acid-free hematoxylin, dehydrated and mounted in Eukitt (O. Kindler $\mathrm{GmbH} \& \mathrm{Co}$, Freiburg, D).

Negative controls were performed using the procedure described above, omitting the first antibody. Furthermore, a normal control section was processed simultaneously with each tissue block. 
Statistical methods

STATS software (Decision Analyst, Inc., USA) was used. Statistical analysis was carried out by the chi-squared test, $p<0.05$ was considered significant.

\section{Results}

Post-operative bowel motility

The retrospective review of patients' medical files demonstrated the following post-operative bowel motility disorders. Enterocolitis occurred in four patients $(8.3 \%)$. These patients each presented one episode of enterocolitis, occurring 1 to 32 months after surgery (mean 13 months, SD 13 months). Persistent constipation was present in 12 patients out of 26 patients who had a minimal 1-year post-operative follow-up $(46 \%)$. Overflow incontinence occurred in seven children $(14.6 \%)$. Three patients presented both overflow incontinence and persistent constipation. Two patients died 9 and 11 months post-operatively respectively, probably of complicated enterocolitis bouts. These results are summarised in Table 1.

\section{Controls}

CD56 immunohistochemistry-labelled ganglion cells were isolated or in groups In the submucous plexus, and in the myenteric plexus were in groups. Nerve fibres were identified throughout the intestinal wall, the most dense innervation being observed in the mucosa and both smooth muscle layers.

PGP 9.5 immunoreactivity was seen on the same neural structures in consecutive sections. Ganglia were identified in both plexuses, and in two specimens isolated ganglion cells were observed in the lamina propria mucosae. These have previously been described in normal human colon by PGP 9.5 immunohistochemistry [26]. Nerve-fibre distribution was identical to that observed with CD56 immunohistochemistry.

c-Kit immunohistochemistry labelled two cell populations. The first comprised fusiform to ovoid cells with large oval nuclei, sparse cytoplasm, and branching processes, corresponding to ICC (Fig. 2). These cells were situated in both the circular and longitudinal muscle layers, and within the myenteric plexus concentrating around the myenteric ganglia.

In the circular muscle layer, the cells were more concentrated at the submucosal border. None of these cells were found within the mucosa or the submucous layer. The second population was composed of numerous rounded cells situated within the mucosa and submucous layer; these are mast cells, which also express the c-Kit receptor. ICC distribution was identical at the hepatic and splenic colon flexures. The intensity of immunostaining under light microscopy was variable between specimens, but cell distribution was identical in all controls confirmed by a review of serial sections.

\section{Resected bowel in Hirschsprung's disease}

Colon innervation of the resected bowel's proximal segment determined by CD56 and PGP 9.5 immunohistology was normal in 27 patients $(56.3 \%)$. Aganglionosis was found in five specimens $(10.4 \%)$, IND B in three specimens $(6.3 \%)$ and hypoganglionosis in six specimens $(12.5 \%)$. Other non-classified dysganglionoses were observed in seven specimens $(14.6 \%)$ that presented myenteric ganglia heterotopias - three within the longitudinal muscle layer, two within the circular muscle layer and two within both smooth muscle layers.

Functional outcome was compared to proximal segment innervation. No statistically significant difference was found in the outcome of bowel function between patients with normal or abnormal patterns of the enteric nervous system. The results of this comparison are summarised in Table 1.

c-Kit immunohistochemistry, performed on the proximal segment of the resected bowel, labelled the same cell populations as in controls. Semi-quantitative assessment showed that ICC were normally distributed in all specimens (Figs. 3 and 4).

These fusiform to ovoid c-Kit immuno-positive cells could be identified in both smooth muscle layers and within the myenteric plexus, aggregating around the

Table 1 Bowel function outcome and proximal segment innervation in 48 patients operated for Hirschsprung's disease. $A G$ aganglionosis, $H G$ hypoganglionosis, IND B intestinal neuronal dysplasia type $\mathrm{B}, U D G$ unclassified dysganglionoses

\begin{tabular}{|c|c|c|c|c|c|c|c|}
\hline \multirow[t]{2}{*}{ Evolution } & \multicolumn{7}{|c|}{ Histology } \\
\hline & Normal & $\mathrm{AG}$ & $\mathrm{HG}$ & IND B & UDG & Total & $\%$ \\
\hline Uncomplicated & 19 & 4 & 3 & 2 & 3 & 31 & 65 \\
\hline Enterocolitis & 2 & & 1 & & 1 & 4 & 8 \\
\hline Constipation & 7 & & 1 & 1 & 3 & 12 & $46^{*}$ \\
\hline Overflow incontinence & 2 & & 2 & 1 & 2 & 7 & 15 \\
\hline Death & 1 & 1 & & & & 2 & 4 \\
\hline Number of patients & 27 & 5 & 6 & 3 & 7 & 48 & \\
\hline
\end{tabular}

Statistical analysis performed by chi-squared test was non-significant $(p>0.10)$

* Percentage calculated on the basis of patients followed for a year or more after surgery (26 patients) 
Fig. 2 Interstitial cells of Cajal (ICC) (stained brown) in a normal colon segment. $C M L$ circular muscle layer, $L M L$ longitudinal muscle layer, *myenteric plexus ganglion (c-Kit immunohistochemistry, $\times 200)$

Fig. 3 Interstitial cells of Cajal (ICC) (stained brown) in an aganglionic colon segment. $C M L$ circular muscle layer, $L M L$ longitudinal muscle layer (c-Kit immunohistochemistry, $\times 200)$

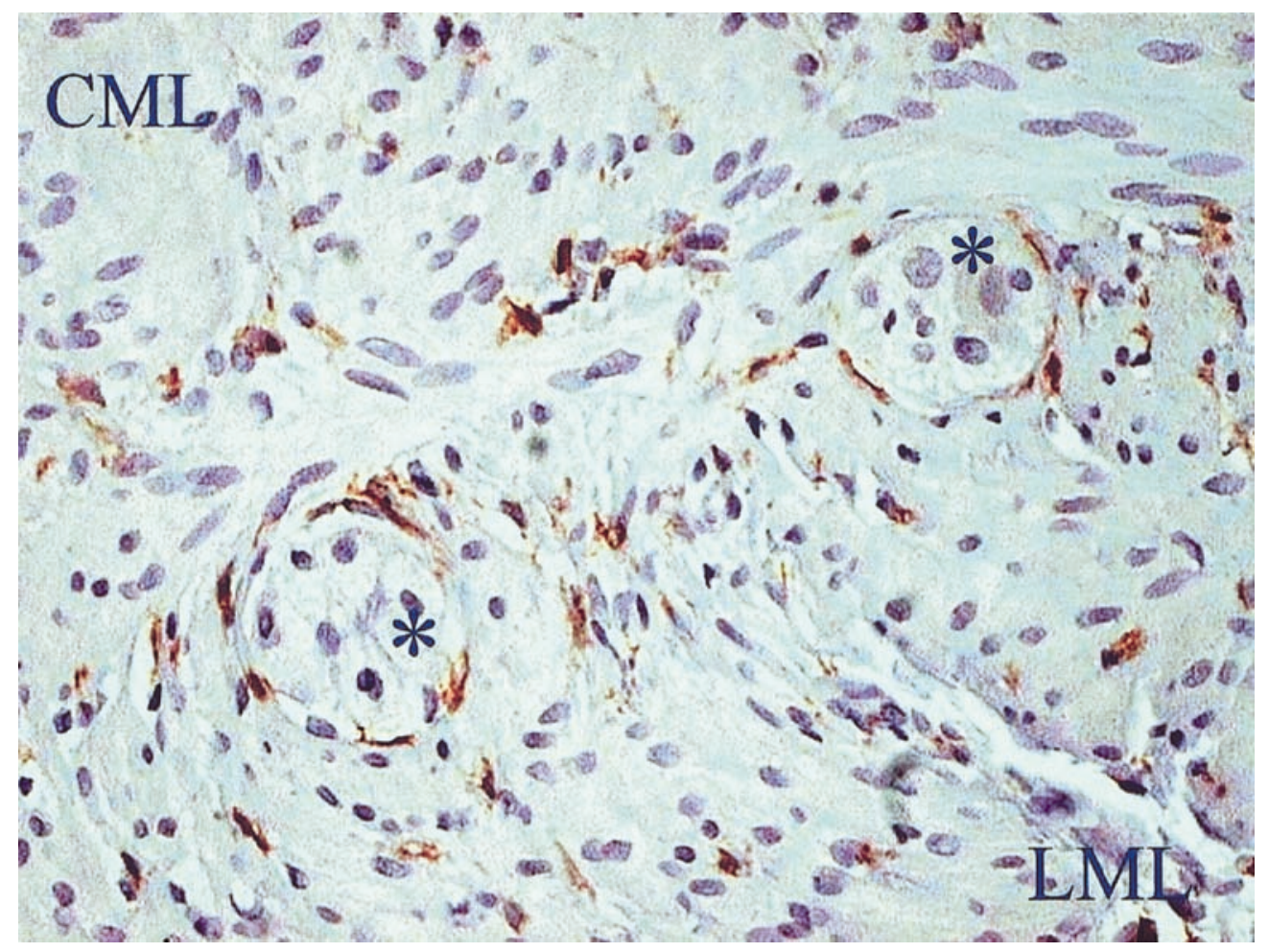

myenteric ganglia in the same concentration as in controls. ICC also concentrated at the submucosal border in the same pattern as in controls. These cells were also present in the myenteric plexus in aganglionic segments where neural elements were absent. None of these cells were present within the mucosa or submucous layer. Intensity of immunostaining presented the same variability as in controls. 
Fig. 4 Interstitial cells of Cajal (ICC) (stained brown) in the circular muscle layer of an aganglionic colon segment (c-Kit immunohistochemistry, $\times 400)$

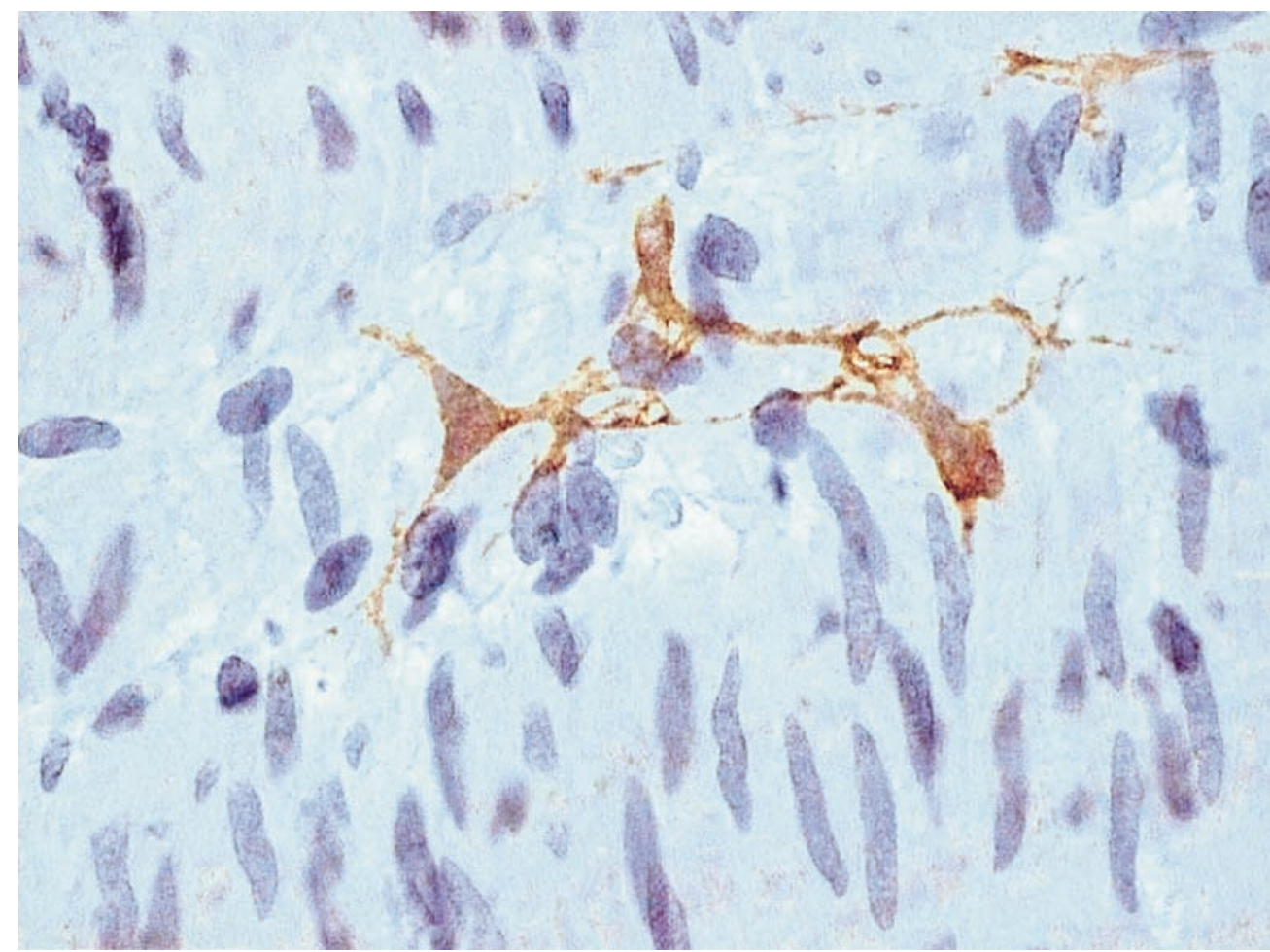

\section{Discussion}

The overall rate of bowel motility disorders after surgery for Hirschsprung's disease ranges between 30 and $45 \%$ in most series $[20,24,3]$. Our data is similar in most respects to previous reports, but the rate of persistent constipation in our patients is consistently higher at $46 \%$, this complication affecting approximately $20 \%$ of patients in precedent reports $[20,24]$. Our patients include $54 \%$ of western African children, referred to our institution through a humanitarian collaboration program. The follow-up of these children is for the main part assured in their home country, and this explains the important variation in the length of post-operative follow-up in our series. Patients from this program who present complicated outcomes are probably overreported to our institution compared to those with an uncomplicated evolution, explaining in part the higher constipation rate in our study. The two patients who died within a year after surgery were included in this program and deceased in their home countries. Reports by local medical teams of abdominal distension and fever before the death of these two children let us believe that they might have succumbed to enterocolitis bouts.

Previous studies have linked abnormal histology of the remaining bowel after pull-through surgery with post-operative bowel function disorders [20, 9, 2]. Recently, Schulten et al. [20] reviewed a series of 101 patients and demonstrated that abnormal histological patterns seem to increase the incidence of bowel motility disorders. One observations of this study was that aganglionosis of the remaining segment implied a diffi- cult post-operative course in all four such cases reported. However, the statistical significance of these observations was not reported. No clear-cut differences between the evolution of patients with normally and abnormally innervated remaining colon emerged from our observations, and of our five patients with proximal segment aganglionosis, only one had a complicated evolution (deceased 9 months post-operatively, probably of complicated enterocolitis). It is also noteworthy that $30 \%$ of patients with normal innervation had a complicated post-operative outcome, among which one child deceased of probable complicated enterocolitis. This demonstrates that normal innervation of the remaining colon does not guarantee a favourable outcome and that factors other than innervation condition the evolution after pull-through surgery. A prospective study comparing clinical outcome with remaining colon innervation is required to further explore their relation.

Among these possible factors, we studied the distribution of ICC. These cells are presumed to generate electrical pacemaker activity in the human gut, although investigations concerning their function have only been conducted in murine tissue $[6,11,23,1]$. c-Kit immunopositivity is believed to identify ICC that are classically identified by electron microscopy [7, 17, 18, 19]. This has been clearly demonstrated in mice and is believed to apply in human gut. The importance of these cells in bowel motility has encouraged research on ICC distribution in Hirschsprung's disease and allied disorders. The results of these studies are contradicting, certain reporting abnormal cell distribution $[28,25,29,7]$ and others the same distribution as in controls [5]. 
In our study, control specimens demonstrated c-Kit immunoreactivity similar to previous descriptions [22, $25,5]$, where ICC were confined to both smooth muscle layers and the myenteric plexus. The use of a polyclonal anti-c-Kit antibody combined with an enhancement method for antigen retrieval allowed us to obtain good quality, reproducible immunostaining on paraffinembedded specimens confirmed by the review of serial sections for each specimen. No regional differences were observed between proximal and distal specimens of the transverse colon, and ICC aggregation around the myenteric plexus and at the submucosal border was observed at both transplant extremities.

Our principal observation was that ICC distribution was normal throughout the whole panel of patients operated for Hirschsprung's disease. Abnormal innervation of the bowel did not disrupt this distribution; aganglionosis, hypoganglionosis, NID B and unclassified dysganglionoses were all associated with normal c-Kit immunohistology. Light and electron microscopy studies in mouse colon have shown that ICC have a nonneural origin, developing from a common precursor with smooth muscle cells [23, 8]. In our observation, ICC seem to develop and differentiate normally independently of neural structures, despite abnormally distributed or absent enteric neurons. Enteric neurons are supposed to produce c-Kit's ligand stem-cell factor in the human gut. This cytokine is known to be essential in the proliferation, migration and differentiation of stem cells, a role that has been well demonstrated in hematopoietic and neural crest-cell lineages. This means an alternative pathway must exist to support the differentiation and development of ICC in the human bowel. Some animal data supports the idea that mammalian ICC are not developmentally dependent on neurons. $\mathrm{Wu}$ et al. [27] showed that ICC differentiation and survival in mice requires stem-cell factor that can be provided by neurons, but also cells in the smooth muscle lineage. The potential role of other cytokines in ICC development has yet to be described.

The normal distribution and morphology of ICC by light microscopy does not exclude modifications or damage at an ultrastructural level. A recent report by Huizinga et al. [7] of a full-term infant without an enteric system showed ultrastructural damage of ICC in the deep-muscular plexus by electron microscopy. However, these same cells were not visible by c-Kit immunohistochemistry. Furthermore, the function of ICC was not assessed in this study. Functional studies of ICC involve recording slow-wave electrical activity at a cellular level. Numerous animal studies of ICC electrophysiology have been performed and could be transposed to human research. The development of techniques for isolation and culture of human ICC should be encouraged. These cultivated cells could then be submitted to patch-clamp experiments and other electrophysiological measurements. Alternatively, macroscopic slow-wave activity could prove to be a proxy indicator of ICC functional integrity. This electrical slow-wave activity can be recorded in human colon surgical specimens [15]. Further studies are necessary to determine if ICC ultrastructure and function are normal in the remaining colon of patients operated for Hirschsprung's disease.

In conclusion, distribution of ICC does not seem to contribute to post-operative bowel dysmotility in patients operated for Hirschsprung's disease. Their distribution was found to be normal in the proximal resected bowel of all the patients we operated. In our study, abnormal innervation is not linked to postoperative complications. Furthermore, ICC apparently develop normally in the human bowel, even when bowel innervation is anomalous.

\section{References}

1. Der-Silaphet T, Malysz J, Hagel S, Arsenault AL, Huizinga JD (1998) Interstitial cells of Cajal direct normal propulsive contractile activity in the mouse small intestine. Gastroenterology 114:724-736

2. Ghose SI, Squire BR, Batcup G, Crabbe DC (2000) Hirschsprung's disease: problems with transition-zone pull-through. J Pediatr Surg 35:1805-1809

3. Hackam DJ, Superina RA, Pearl RH (1997) Single-stage repair of Hirschsprung's disease: a comparison of 109 patients over 5 years. J Pediatr Surg 32:1028-1032

4. Holschneider AM, Meier-Ruge W, Ure BM (1993) Hirschsprung's disease and allied disorders - a review. Eur J Pediatr Surg 4:260-266

5. Horisawa M, Watanabe Y, Torihashi S (1998) Distribution of c-Kit immunopositive cells in normal human colon and in Hirschsprung's disease. J Pediatr Surg 33:1209-1214

6. Huizinga JD, Thuneberg L, Klüppel M, Malysz J, Mikkelsen HB, Bernstein A (1995) W/kit gene required for interstitial cells of Cajal and for intestinal pacemaker activity. Nature 373:347349

7. Huizinga JD, Berezin I, Sirkar K, Hewlett B, Donnelly G, Bercik P, Ross C, Algoufi T, Fitzgerald P, Der T, Riddell RH, Collins SM, Jacobson K (2001) Development of interstitial cells of Cajal in a full-term infant without an enteric nervous system. Gastroenterology 120:561-7

8. Klüppel M, Huizinga JD, Malysz J, Bernstein A (1998) Developmental origin and Kit-dependent development of the interstitial cells of Cajal in the mammalian small intestine. Dev Dynam 211:60-71

9. Kobayashi H, Hirakawa H, Surana R, O'Briain DS, Puri P (1995) Intestinal neuronal dysplasia is a possible cause of persistent bowel symptoms after pull-through operation for Hirschsprung's disease. J Pediatr Surg 30:253-259

10. Krammer HJ, Karahan ST, Sigge W, Kühnel W (1993) Immunohistochemistry of markers of the enteric nervous system in whole-mount preparations of the human colon. Eur $\mathbf{J}$ Pediatr Surg 4:274-278

11. Maeda H, Yamagata A, Nishikawa S, Yoshinaga K, Kobayashi S, Nishi K, Nishikawa S (1992) Requirement of c-kit for development of intestinal pacemaker system. Development 116:369-375

12. Mechtersheimer G, Staudter M, Möller P (1991) Expression of the natural killer-cell associated antigens CD56 and CD57 in human neural and striated muscle cells and their tumors. Cancer Res 51:1300-1307

13. Meier-Ruge W, Gambazzi F, Kaüfeler RE, Schmid P, Schmidt CP (1994) The neuropathological diagnosis of neuronal intestinal dysplasia (NID B). Eur J Pediatr Surg 4:267-273

14. Meyrat BJ, Lesbros Y, Laurini RN (2001) Assessment of the colon innervation with serial biopsies above the aganglionic 
zone before the pull-through procedure in Hirschsprung's disease. Pediatr Surg Int 17:129-135

15. Rae MG, Fleming N, McGregor DB, Sanders KM, Keef KD (1998) Control of motility patterns in the human colonic circular muscle layer by pacemaker activity. J Physiol 510:309-20

16. Romanska HM, Bishop AE, Brereton RJ, Spitz L, Polak JM (1993) Increased expression of muscular neural cell adhesion molecule in congenital aganglionosis. Gastroenterology 105:1104-1109

17. Rumessen JJ, Thuneberg L (1991) Interstitial cells of Cajal in human small intestine. Ultrastructural identification and organization between the main smooth muscle layers. Gastroenterology 100:1417-1431

18. Rumessen JJ, Thuneberg L (1996) Pacemaker cells in the gastrointestinal tract: interstitial cells of Cajal. Scand J Gastroenterol 31:82-94

19. Sanders MS (1996) A case for interstitial cells of Cajal as pacemakers and mediators of neurotransmission in the gastrointestinal tract. Gastroenterology 111:492-515

20. Schulten D, Holschneider AM, Meier-Ruge W (2000) Proximal segment histology of resected bowel in Hirschsprung's disease predicts postoperative bowel function. Eur J Pediatr Surg 10:378-381

21. Shi SR, Key ME, Kalra KL (1991) Antigen retrieval in formalin-fixed, paraffin-embedded tissues: an enhancement method for immunohistochemical staining based on microwave oven heating of tissue sections. J Histochem Cytochem 39:741-748
22. Torihashi S, Ward SM, Sanders KM (1997) Development of ckit-positive cells and the onset of electrical rhythmicity in murine small intestine. Gastroenterology 112:144-155

23. Torihashi S, Horisawa M, Watanabe Y (1999) c-Kit immunoreactive interstitial cells in the human gastrointestinal tract. J Auton Nerv Syst 75:38-50

24. Ure BM, Holschneider AM, Meier-Ruge W (1993) Neuronal intestinal malformations: a retro- and prospective study on 203 patients. Eur J Pediatr Surg 4:279-286

25. Vanderwinden JM, Rumessen JJ, Liu H, Descamps D, De Laet MH, Vanderhaeghen JJ (1996) Interstitial cells of Cajal in human colon and in Hirschsprung's disease. Gastroenterology 111:901-910

26. Wedel T, Roblick U, Gleiss J, Schiedeck T, Bruch HP, Kuhnel W, Krammer HJ (1999) Organization of the enteric nervous system in the human colon demonstrated by wholemount immunohistochemistry with special reference to the submucous plexus. Anat Anz 181:327-337

27. Wu JJ, Rothman TP, Gershon MD (2000) Development of the interstitial cell of Cajal: origin, kit dependence and neuronal and nonneuronal sources of kit ligand. J Neurosci Res 59:384-401

28. Yamataka A, Kato Y, Tibboel D, Murata Y, Sueyoshi N, Fujimoto T, Nishiye H, Miyano T (1995) A lack of intestinal pacemaker (c-kit) in aganglionic bowel of patients with Hirschsprung's disease. J Pediatr Surg 30:441-444.

29. Yamataka A, Ohshiro K, Kobayashi H, Fujiwara T, Sunagawa M, Miyano T (1997) Intestinal pacemaker C-KIT ${ }^{+}$cells and synapses in allied Hirschsprung's disorders. J Pediatr Surg 32:1069-1074 